\title{
论 文
}

\section{页岩气纳米孔真实气体传输模型}

\section{吴克柳 ${ }^{\mathbb{Q}(* *}$ ，李相方 ${ }^{\circledR}$, CHEN ZhangXin $^{(2)}$}

(1) 中国石油大学(北京)石油工程学院, 北京 102249 ;

(2) Department of Chemical and Petroleum Engineering, University of Calgary, Calgary T2N1N4, Canada

*E-mail: wukeliu19850109@163.com

收稿日期: 2015-06-05; 接受日期: 2015-08-29

国家自然科学基金重大项目(批准号: 51490654)、国家自然科学基金(批准号: 51374222)和国家科技重大专项(编号: 2011ZX05030-005-04) 资助

摘要页岩富含纳米孔, 纳米孔气体传输不同于宏观流体流动. 基于滑脱流动和克努森扩散两种传输机理, 分别以分子之间碰撞频率和分子与壁面碰撞频率占总碰撞频率的比值作为滑脱流动和克努森扩散的权重系数, 耦合这两种机理，建立了理想气体传输模型. 同时考虑高压条件下真实气体分子间相互作用力和气体分子自身 体积对气体传输的影响, 建立了页岩纳米孔真实气体传输模型. 模型可靠性通过分子模拟结果验证. 结果表明: 纳米孔真实气体传输模型能够更合理地描述所有的气体传输机理, 包括连续流动、滑脱流动和过渡流动; 真实气 体效应对气体传输的影响可高达 $23 \%$, 其受压力、温度、纳米孔尺度和气体类型的控制; 在室内实验条件下模拟 页岩纳米孔气体传输时, 用氦气代替甲烷，低估了甲烷的传输能力 $65.09 \%$; 用氮气代替甲烷，高估了甲烷的传 输能力 $106.27 \%$.

关键词页岩气, 纳米孔, 真实气体, 滑脱流动, 克努森扩散

\section{1 引言}

由于吸附分离、催化、给药和页岩气开发的快速 发展, 纳米孔气体传输模拟变得越来越重要 ${ }^{[1 \sim 3]}$. 页 岩富含纳米孔，直径多为几纳米至几十纳米 ${ }^{[4,5]}$. 页 岩纳米孔气体传输模拟是页岩气准确数值模拟的基 础 $^{[6]}$, 对页岩气生产动态预测和压裂参数优化设计具 有重要的指导意义 ${ }^{[7,8]}$.

典型页岩气条件下(克努森数为 0.0002 6), 纳米 孔气体传输机理包括连续流动、滑脱流动和过渡流
动 ${ }^{[9]}$, 现有模型很难描述所有的传输机理. Klinkenberg $^{[10]}$ 模型仅适用于连续流动和滑脱流动; 尘气模 型将扩散量和水动力流量任意叠加, 未考虑两者的 耦合 ${ }^{[11]}$; Beskok 和 Karniadakis ${ }^{[12]}$ 模型虽能描述所有 的传输机理, 但仅适合克努森数小于等于 1 情况, 且 含有经验系数; Javadpour ${ }^{[13]}$ 模型为滑脱流动和克努 森扩散的线性相加，未考虑两者的耦合; Anderson 等 人 $^{[14]}$ 提出的模型也能描述所有的传输机理, 但含经 验系数, 需实验数据确定. 因此, 急需开发出简单的, 且能描述所有传输机理的纳米孔气体传输模型.

引用格式: 吴克柳, 李相方, Chen Z X. 页岩气纳米孔真实气体传输模型. 中国科学: 技术科学, 2016, 46: 68-78

Wu K L, Li X F, Chen Z X. Real gas transport through nanopores of shale gas reservoirs (in Chinese). Sci Sin Tech, 2015, 45: 68-78, doi: 10.1360/N092015-00076 
统计数据表明, 北美页岩气藏埋深普遍为 500 $3000 \mathrm{~m}$, 地层压力为 $5 \sim 30 \mathrm{MPa}^{[15]}$. 高压条件下页岩 纳米孔气体传输机理和相对低压条件下的吸附分离、 催化和给药的纳米孔气体传输机理是不同的 ${ }^{[16]}$. 高 压条件下, 气体稠密, 范德华力增大, 气体分子之间 的相互作用力对气体传输不可忽略; 而且在纳米孔 狭窄的空间中, 气体分子不再是一个点, 需考虑分子 自身体积对气体传输的影响 ${ }^{[17]}$. Wang 等人 ${ }^{[18]}$ 通过蒙 特卡罗直接模拟法研究微纳米尺度气体传输机理, 在开度为 $10 \mathrm{~nm}$ 和压力为 $4.4 \mathrm{MPa}$ 条件下, 与理想气 体相比, 考虑真实气体效应的气体传输能力增加了 28.63\%; Michel 等人 ${ }^{[2]}$ 研究也表明, 在纳米孔半径 5 $\mathrm{nm}$ 和 $99.97 \mathrm{MPa}$ 条件下, 考虑真实气体效应的页岩 气体传输能力增强了 $60 \%$; $\mathrm{Ma}$ 等人 ${ }^{[3]}$ 基于 Javadpour 模型 ${ }^{[13]}$, 考虑真实气体效应的页岩气体传输能力提 高了 $10 \%$. 因此, 真实气体效应对页岩纳米孔气体传 输的影响是不可忽略的.

气体黏度、偏差因子和平均分子自由程是温度和 压力的函数. 将考虑真实气体效应的这些宏观参数 代入气体传输模型中, 就能刻画出高压条件下纳米 孔真实气体分子之间相互作用力和自身体积大小对 气体传输的影响. 本文首先基于纳米孔理想气体传 输模型, 考虑真实气体效应的影响, 建立了纳米孔真 实气体传输模型; 并用公开发表的分子模拟数据验 证模型; 然后对计算的结果进行分析和讨论; 最后给 出结论.

\section{2 模型建立}

基于滑脱流动和克努森扩散两种传输机理, 分 别以分子之间碰撞频率和分子与壁面碰撞频率占总 碰撞频率的比值作为滑脱流动和克努森扩散的权重 系数, 耦合这两种机理, 建立了气体传输模型. 该模 型可以合理地描述页岩纳米孔气体所有的传输机理, 包括连续流动、滑脱流动和过渡流动.

\section{1 理想气体传输模型}

理想气体平均分子自由程可表达为 ${ }^{[19]}$

$$
\lambda_{\mathrm{i}}=\frac{\eta}{p} \sqrt{\frac{\pi R T}{2 M}},
$$

式中, $\lambda_{\mathrm{i}}$ 是理想气体平均分子自由程, $\mathrm{m} ; \eta$ 是理想气 体黏度, $\mathrm{Pa} \mathrm{s} ; p$ 是压力, $\mathrm{Pa} ; R$ 是气体常数, $\mathrm{J} /(\mathrm{mol} \mathrm{K}) ; T$
是地层温度, $\mathrm{K} ; M$ 是气体摩尔质量, $\mathrm{kg} / \mathrm{mol}$.

纳米孔理想气体对应的克努森数可表达为 ${ }^{[20]}$

$$
K n_{\mathrm{i}}=\frac{\lambda_{\mathrm{i}}}{2 r},
$$

式中, $K n_{\mathrm{i}}$ 是理想气体对应的克努森数, 无因次; $r$ 是 孔隙半径, $\mathrm{m}$.

\subsection{1 滑脱流动}

当克努森数 $10^{-3}<K n_{\mathrm{i}}<10^{-1}$, 气体分子间碰撞为 主, 气体传输为滑脱流动. 则考虑滑脱效应和稀有效 应的理想气体滑脱流动可表达为 ${ }^{[21]}$

$$
J_{\text {vsi }}=-\zeta_{\mathrm{mb}} \frac{r^{2} p M}{8 \eta R T}\left(1+\alpha_{\mathrm{i}} K n_{\mathrm{i}}\right)\left(1+\frac{4 K n_{\mathrm{i}}}{1-b K n_{\mathrm{i}}}\right) \frac{\mathrm{d} p}{\mathrm{~d} l},
$$

其中,

$$
\alpha_{\mathrm{i}}=\alpha_{\mathrm{o}} \frac{2}{\pi} \tan ^{-1}\left(\alpha_{1} K n_{\mathrm{i}}^{\beta}\right),
$$

式中 $J_{v s i}$ 是理想气体滑脱流动质量流量, $\mathrm{kg} /\left(\mathrm{m}^{2} \mathrm{~s}\right) ; \alpha_{\mathrm{i}}$ 是理想气体稀有效应系数, 无因次; $b$ 是气体滑脱常 数, 无因次; $l$ 是气体传输方向的距离, $\mathrm{m} ; \alpha_{0}$ 是 $K n \rightarrow$ $\infty$ 时的稀有效应系数, 无因次; $\alpha_{1}$ 是拟合常数, 无因 次; $\beta$ 是拟合常数, 无因次; $\xi_{\mathrm{mb}}=\Phi / \tau$, 气体在多孔介质 中流动时的修正系数, 无因次, 具体推导可参考文献 [9]; $\Phi$ 是孔隙度, 小数; $\tau$ 是迂曲度, 无因次.

\subsection{2 克努森扩散}

当克努森数 $K n_{\mathrm{i}} \gg 1$, 气体分子与壁面碰撞为主, 气体传输为克努森扩散. 则考虑壁面粗粘度的理想 气体克努森扩散可表达为 ${ }^{[22,23]}$

$$
J_{\mathrm{ki}}=-\frac{2}{3} \zeta_{\mathrm{mb}} r \delta^{D_{\mathrm{f}}-2}\left(\frac{8 M}{\pi R T}\right)^{0.5} \frac{\mathrm{d} p}{\mathrm{~d} l},
$$

式中, $J_{\mathrm{ki}}$ 是理想气体克努森扩散质量流量, $\mathrm{kg} /\left(\mathrm{m}^{2} \mathrm{~s}\right)$; $\sigma$ 是分子直径 $d_{\mathrm{M}}$ 与局部孔隙直径 $2 r$ 的比值, 无因次; $D_{\mathrm{f}}$ 是孔隙壁面分形维数, 无因次.

\subsection{3 总传输量}

分别以分子之间碰撞频率和分子与壁面碰撞频 率占总碰撞频率的比值作为滑脱流动和克努森扩 散的权重系数, 则纳米孔理想气体总传输量可表达 为 ${ }^{[9]}$

$$
J_{\mathrm{ti}}=\omega_{\mathrm{vsi}} J_{\mathrm{vsi}}+\omega_{\mathrm{ki}} J_{\mathrm{ki}},
$$


式中, $\omega_{\mathrm{vsi}}=\frac{1}{\left(1+K n_{\mathrm{i}}\right)} ; \omega_{\mathrm{ki}}=\frac{1}{\left(1+1 / K n_{\mathrm{i}}\right)} \cdot J_{\mathrm{ti}}$ 是理想气 体总质量流量, $\mathrm{kg} /\left(\mathrm{m}^{2} \mathrm{~s}\right) ; \omega_{\mathrm{vsi}}$ 是理想气体滑脱流动权 重系数, 无因次; $\omega_{\mathrm{ki}}$ 是理想气体克努森扩散权重系 数, 无因次.

将(3)式、(4)式代入(5)式, 得:

$$
\begin{aligned}
J_{\mathrm{ti}}= & -\frac{1}{\left(1+K n_{\mathrm{i}}\right)} \zeta_{\mathrm{mb}} \frac{r^{2} p M}{8 \eta R T}\left(1+\alpha_{\mathrm{i}} K n_{\mathrm{i}}\right)\left(1+\frac{4 K n_{\mathrm{i}}}{1-b K n_{\mathrm{i}}}\right) \frac{\mathrm{d} p}{\mathrm{~d} l} \\
& -\frac{1}{\left(1+1 / K n_{\mathrm{i}}\right)} \frac{2}{3} \zeta_{\mathrm{mb}} r \delta^{D_{\mathrm{f}}-2}\left(\frac{8 M}{\pi R T}\right)^{0.5} \frac{\mathrm{d} p}{\mathrm{~d} l} .
\end{aligned}
$$

根据(6)式, 则对应的纳米孔理想气体传导率为

$$
\begin{aligned}
C_{\mathrm{ti}}= & \frac{1}{\left(1+K n_{\mathrm{i}}\right)} \zeta_{\mathrm{mb}} \frac{r^{2} p M}{8 \eta R T}\left(1+\alpha_{\mathrm{i}} K n_{\mathrm{i}}\right)\left(1+\frac{4 K n_{\mathrm{i}}}{1-b K n_{\mathrm{i}}}\right) \\
& +\frac{1}{\left(1+1 / K n_{\mathrm{i}}\right)} \frac{2}{3} \zeta_{\mathrm{mb}} r \delta^{D_{\mathrm{f}}-2}\left(\frac{8 M}{\pi R T}\right)^{0.5},
\end{aligned}
$$

式中, $C_{\mathrm{ti}}$ 是理想气体总传导率, $\mathrm{s}$.

当克努森数 $K n_{\mathrm{i}}<10^{-3},(6)$ 式退化为

$$
J_{\mathrm{vi}}=-\zeta_{\mathrm{mb}} \frac{r^{2} p M}{8 \eta R T} \frac{\mathrm{d} p}{\mathrm{~d} l},
$$

可描述连续流动, 其中 $J_{\mathrm{vi}}$ 是理想气体连续流质量流 量, $\mathrm{kg} /\left(\mathrm{m}^{2} \mathrm{~s}\right)$; 当克努森数 $10^{-3}<K n_{\mathrm{i}}<10^{-1}$, (6)式退化 为(3)式, 可描述滑脱流动; 当克努森数 $K n_{\mathrm{i}} \gg 1,(6)$ 式 退化为 (4) 式, 可描述克努森扩散; 当克努森数 $10^{-1}<K n_{\mathrm{i}}<1$, 气体分子间碰撞和气体分子与壁面碰撞 相当, 对气体传输影响均不可忽略, 此条件下的传输 机理为过渡流动, 可用(6)式描述.

\section{2 真实气体传输模型}

页岩纳米孔高压条件下，气体分子间相互作用 力和气体分子自身体积对气体传输的影响不可忽略. 这种真实气体效应的影响可通过真实气体偏差因子、 黏度和平均分子自由程对气体传输的影响来描述.

气体偏差因子是温度和压力的函数, 可通过对 比温度和对比压力表达为 ${ }^{[24]}$

$$
Z=1+\frac{p_{\mathrm{r}}}{10.24 T_{\mathrm{r}}}\left[2.16 \frac{1}{T_{\mathrm{r}}}\left(\frac{1}{T_{\mathrm{r}}}+1\right)-1\right],
$$

式中 $p_{\mathrm{r}}=p / p_{\mathrm{c}} ; T_{\mathrm{r}}=T / T_{\mathrm{c}} ; Z$ 是气体偏差因子, 无因 次; $p_{\mathrm{r}}$ 是气体对比压力, 无因次; $T_{\mathrm{r}}$ 是气体对比温度, 无 因次; $p_{\mathrm{c}}$ 是气体临界压力, $\mathrm{MPa} ; T_{\mathrm{c}}$ 是气体临界温度, $\mathrm{K}$.
同样, 气体黏度也是温度和压力的函数. 任意条 件下的气体黏度用对比温度和对比压力表达为 ${ }^{[25]}$

$$
\eta_{\mathrm{r}}=\eta\left[1+\frac{A_{1}}{T_{\mathrm{r}}^{5}}\left(\frac{p_{\mathrm{r}}^{4}}{T_{\mathrm{r}}^{20}+p_{\mathrm{r}}^{4}}\right)+A_{2}\left(\frac{p_{\mathrm{r}}}{T_{\mathrm{r}}}\right)^{2}+A_{3}\left(\frac{p_{\mathrm{r}}}{T_{\mathrm{r}}}\right)\right],
$$

式中, $\eta_{\mathrm{r}}$ 是真实气体黏度, Pa $\mathrm{s} ; A_{1}, A_{2}$ 和 $A_{3}$ 均为拟合 常数, 无因次.

真实气体平均分子自由程可表达为 ${ }^{[26]}$

$$
\lambda_{\mathrm{r}}=\frac{\eta_{\mathrm{r}}}{p} \sqrt{\frac{\pi Z R T}{2 M}},
$$

式中, $\lambda_{\mathrm{r}}$ 是真实气体平均分子自由程, $\mathrm{m}$.

纳米孔真实气体对应的克努森数可写为

$$
K n_{\mathrm{r}}=\frac{\lambda_{\mathrm{r}}}{2 r},
$$

式中, $K n_{\mathrm{r}}$ 是真实气体对应的克努森数, 无因次.

\subsection{1 滑脱流动}

真实气体滑脱流动可表达为

$$
J_{\mathrm{vsr}}=-\zeta_{\mathrm{mb}} \frac{r^{2} M}{8 \eta_{\mathrm{r}} R T} \frac{p}{Z}\left(1+\alpha_{\mathrm{r}} K n_{\mathrm{r}}\right)\left(1+\frac{4 K n_{\mathrm{r}}}{1-b K n_{\mathrm{r}}}\right) \frac{\mathrm{d} p}{\mathrm{~d} l},
$$

式中 $\alpha_{\mathrm{r}}=\alpha_{\mathrm{o}} \frac{2}{\pi} \tan ^{-1}\left(\alpha_{1} K n_{\mathrm{r}}{ }^{\beta}\right) ; J_{\mathrm{vsr}}$ 是真实气体滑脱流 动质量流量, $\mathrm{kg} /\left(\mathrm{m}^{2} \mathrm{~s}\right) ; \alpha_{\mathrm{r}}$ 是真实气体稀有效应系数, 无因次.

结合(8)式、(9)式、(11)式和(12)式可计算真实气 体滑脱流动量.

\subsection{2 克努森扩散}

真实气体克努森扩散可表达为

$$
J_{\mathrm{kr}}=-\frac{2}{3} \zeta_{\mathrm{mb}} r \delta^{D_{\mathrm{f}}-2}\left(\frac{8 Z M}{\pi R T}\right)^{0.5} \frac{p}{Z} C_{\mathrm{g}} \frac{\mathrm{d} p}{\mathrm{~d} l},
$$

式中 $C_{\mathrm{g}}=\frac{1}{p}-\frac{1}{Z} \frac{\mathrm{d} Z}{\mathrm{~d} p} ; J_{\mathrm{kr}}$ 是真实气体克努森扩散质量 流量, $\mathrm{kg} /\left(\mathrm{m}^{2} \mathrm{~s}\right) ; C_{\mathrm{g}}$ 是气体压缩因子, $1 / \mathrm{MPa}$. 结合 $(8)$ 和(13)式即可获得真实气体克努森扩散量.

\subsection{3 总传输量}

与理想气体一样, 结合(12)和(13)式，并分别以 分子之间的碰撞频率和分子与壁面的碰撞频率占总 碰撞频率的比值作为滑脱流动和克努森扩散的权重 
系数, 则纳米孔真实气体总传输量可表达为

$$
\begin{aligned}
J_{\mathrm{tr}}= & -\frac{1}{\left(1+K n_{\mathrm{r}}\right)} \zeta_{\mathrm{mb}} \frac{r^{2} p M}{8 \eta_{\mathrm{r}} R T Z}\left(1+\alpha_{\mathrm{r}} K n_{\mathrm{r}}\right)\left(1+\frac{4 K n_{\mathrm{r}}}{1-b K n_{\mathrm{r}}}\right) \frac{\mathrm{d} p}{\mathrm{~d} l} \\
& -\frac{1}{\left(1+1 / K n_{\mathrm{r}}\right)} \frac{2}{3} \zeta_{\mathrm{mb}} r \delta^{D_{\mathrm{f}}-2}\left(\frac{8 Z M}{\pi R T}\right)^{0.5} \frac{p}{Z} C_{\mathrm{g}} \frac{\mathrm{d} p}{\mathrm{~d} l},
\end{aligned}
$$

式中 $J_{\mathrm{tr}}$ 是真实气体总质量流量, $\mathrm{kg} /\left(\mathrm{m}^{2} \mathrm{~s}\right)$.

根据(14)式, 则对应的纳米孔真实气体传导率为

$$
\begin{aligned}
C_{\mathrm{tr}}= & \frac{1}{\left(1+K n_{\mathrm{r}}\right)} \zeta_{\mathrm{mb}} \frac{r^{2} p M}{8 \eta_{\mathrm{r}} R T Z}\left(1+\alpha_{\mathrm{r}} K n_{\mathrm{r}}\right)\left(1+\frac{4 K n_{\mathrm{r}}}{1-b K n_{\mathrm{r}}}\right) \\
& +\frac{1}{\left(1+1 / K n_{\mathrm{r}}\right)} \frac{2}{3} \zeta_{\mathrm{mb}} r \delta^{D_{\mathrm{r}}-2}\left(\frac{8 Z M}{\pi R T}\right)^{0.5} \frac{p}{Z} C_{\mathrm{g}},
\end{aligned}
$$

式中 $C_{\mathrm{tr}}$ 是真实气体总传导率, $\mathrm{s}$.

与理想气体总传输量(6)式类似, 真实气体总传 输量(14)式也可描述连续流动、滑脱流动、过渡流动 和克努森扩散.

\section{3 模型验证}

由于分子模拟数据较准确, 因此常用来验证纳 米尺度气体传输模型; 相反, 实验往往不能准确地反 映理论模型的理想条件, 因此较少用来验证纳米尺 度气体传输模型 ${ }^{[27,28]}$. 因此, 本文将模型计算结果与 公开发表的分子模拟结果进行对比, 验证模型的可 靠性. 模型计算参数见表 1 .

\section{表 1 计算参数总结表}

\begin{tabular}{cccc}
\hline 参数名 & 符号 & 单位 & 数值 \\
\hline 孔隙度 & $\Phi$ & 小数 & 0.05 \\
孔隙迂曲度 & $\tau$ & 无因次 & 4.3 \\
孔隙半径 & $r$ & $\mathrm{~m}$ & $2.0 \times 10^{-9} \sim 1.0 \times 10^{-6}$ \\
气体常数 & $R$ & $\mathrm{~J} /(\mathrm{mol} \mathrm{K})$ & 8.314462 \\
温度 & $T$ & $\mathrm{~K}$ & $423 / 298$ \\
$K \mathrm{n} \rightarrow \infty$ 时稀有效应系数 & $\alpha_{0}$ & 无因次 & 1.19 \\
拟合常数 & $\alpha_{1}$ & 无因次 & 4.0 \\
拟合常数 & $\beta$ & 无因次 & 0.4 \\
气体滑脱常数 & $b$ & 无因次 & -1 \\
分子直径与局部孔隙直径 & $\sigma$ & 无因次 & 0.5 \\
的比值 & & 无因次 & 2.5 \\
孔隙壁面分形维数 & $D_{\mathrm{f}}$ & 无因次 & 7.9 \\
拟合常数 & $A_{1}$ & 无因次 & $9.0 \times 10^{-6}$ \\
拟合常数 & $A_{2}$ & 无因次 & 0.28 \\
拟合常数 & $A_{3}$ & 无 &
\end{tabular}

\section{1 理想气体传输模型验证}

为了对比方便, 分别以连续流流量和克努森扩 散量为基础, 将纳米孔理想气体总传输量无因次化, 分别表达为

$$
\begin{aligned}
\frac{J_{\mathrm{ti}}}{J_{\mathrm{vi}}}=\frac{\left(1+\alpha_{\mathrm{i}} K n_{\mathrm{i}}\right)}{\left(1+K n_{\mathrm{i}}\right)}\left(1+\frac{4 K n_{\mathrm{i}}}{1-b K n_{\mathrm{i}}}\right)+\frac{\delta^{D_{\mathrm{f}}-2} K n_{\mathrm{i}}}{\left(1+1 / K n_{\mathrm{i}}\right)} \frac{128}{3 \pi}, & \\
\frac{J_{\mathrm{ti}}}{J_{\mathrm{ki}}}= & \frac{\left(1+\alpha_{\mathrm{i}} K n_{\mathrm{i}}\right)}{\left(1+K n_{\mathrm{i}}\right)}\left(1+\frac{4 K n_{\mathrm{i}}}{1-b K n_{\mathrm{i}}}\right) \frac{3 \pi}{128 \delta^{D_{\mathrm{r}}-2} K n_{\mathrm{i}}} \\
& +\frac{1}{\left(1+1 / K n_{\mathrm{i}}\right)},
\end{aligned}
$$

式中 $J_{\mathrm{ti}} / J_{\mathrm{vi}}$ 是以连续流流量为基础的无因次理想气体 传输量, 无因次; $J_{\mathrm{ti}} / J_{\mathrm{ki}}$ 是以克努森扩散量为基础的无 因次理想气体传输量, 无因次.

图 1 中“分子模拟”低压稀薄气体传输的数据源 自 Loyalka 和 Hamoodi ${ }^{[29]}$. 由图 1 可知, 本文模型解 析解与分子模拟结果吻合很好, 表明建立的理想气 体传输模型在模拟低压条件下纳米孔稀薄气体的传 输是可靠的.

\section{2 真实气体传输模型验证}

为了验证方便, 用真实气体传输模型计算结果
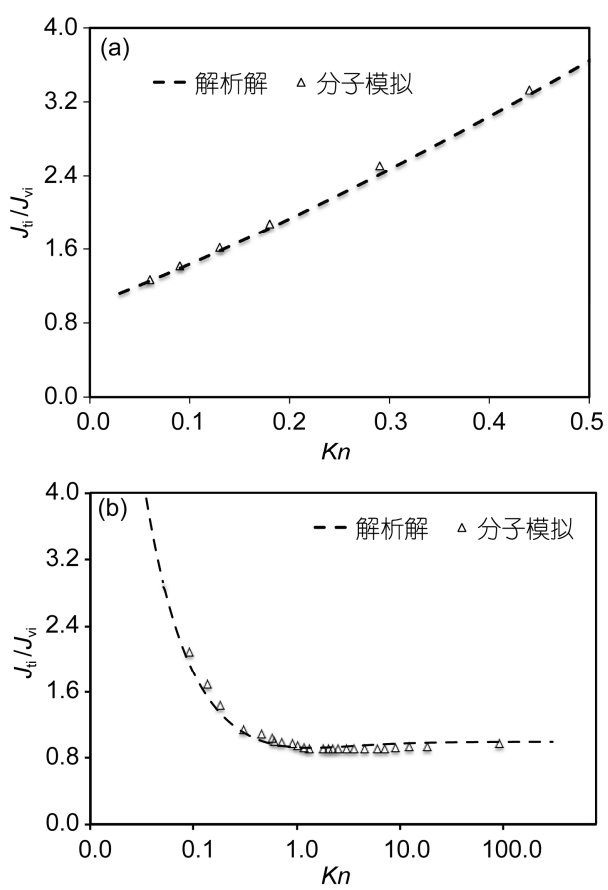

图 1 模型解析解与分子模拟的无因次理想气体传输量 对比 


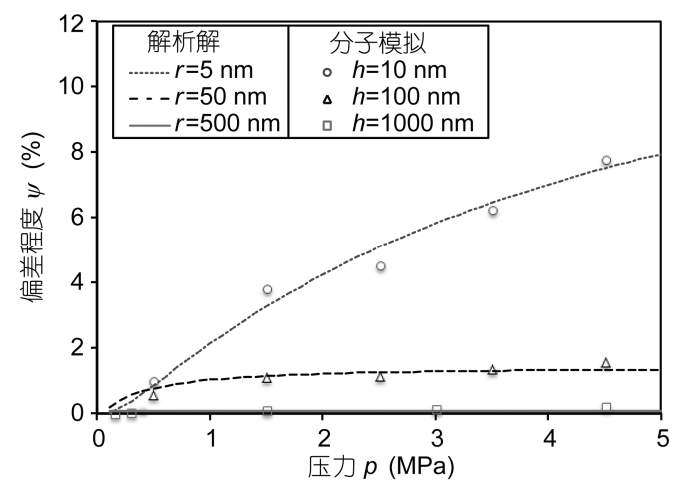

图 2 模型解析解与分子模拟的偏差程度对比

与理想气体传输模型计算结果的偏差程度与分子模 拟的相应结果进行对比. 偏差程度可表达为

$$
\psi=\left(J_{\mathrm{tr}}-J_{\mathrm{ti}}\right) \times 100 \% / J_{\mathrm{ti}},
$$

式中 $\Psi$ 是真实气体传输量与理想气体传输量的偏差 程度, \%.

图 2 中“分子模拟”数据是 Wang 和 $\mathrm{Li}^{[16,17]}$ 用蒙特 卡罗直接模拟的. 由图 2 可见, 模型解析解与分子模 拟结果吻合很好, 表明建立的真实气体传输模型 在模拟高压条件下微纳米尺度真实气体的传输是可 靠的.

\section{4 结果与讨论}

\section{1 纳米孔气体传输}

纳米孔气体传输机理包括连续流动、滑脱流动和 过渡流动. 高压条件下, 气体分子之间碰撞为主, 气 体传输为滑脱流动, 且气体滑脱流动传导率随压力 的增加而增大(图 3(a)), 这是由于气体滑脱流动传导 率本身随压力增加而增大, 而且气体滑脱流动的权 重系数也随压力增加而增大; 低压条件下, 气体分子 与壁面碰撞为主, 气体传输为克努森扩散, 且气体克 努森扩散传导率随压力的降低而增大(图 3(b)), 这是 由于压力对气体克努森扩散传导率不影响, 但克努 森扩散的权重系数却随压力降低而急剧增大. 因此, 随压力降低, 纳米孔气体总传导率先降低后增大(图 $3(\mathrm{c})$ ), 这也是页岩气井开发具有很长稳产期的原因 之一.

\section{2 偏差程度分析}

气体分子之间的相互作用力和气体分子自身体
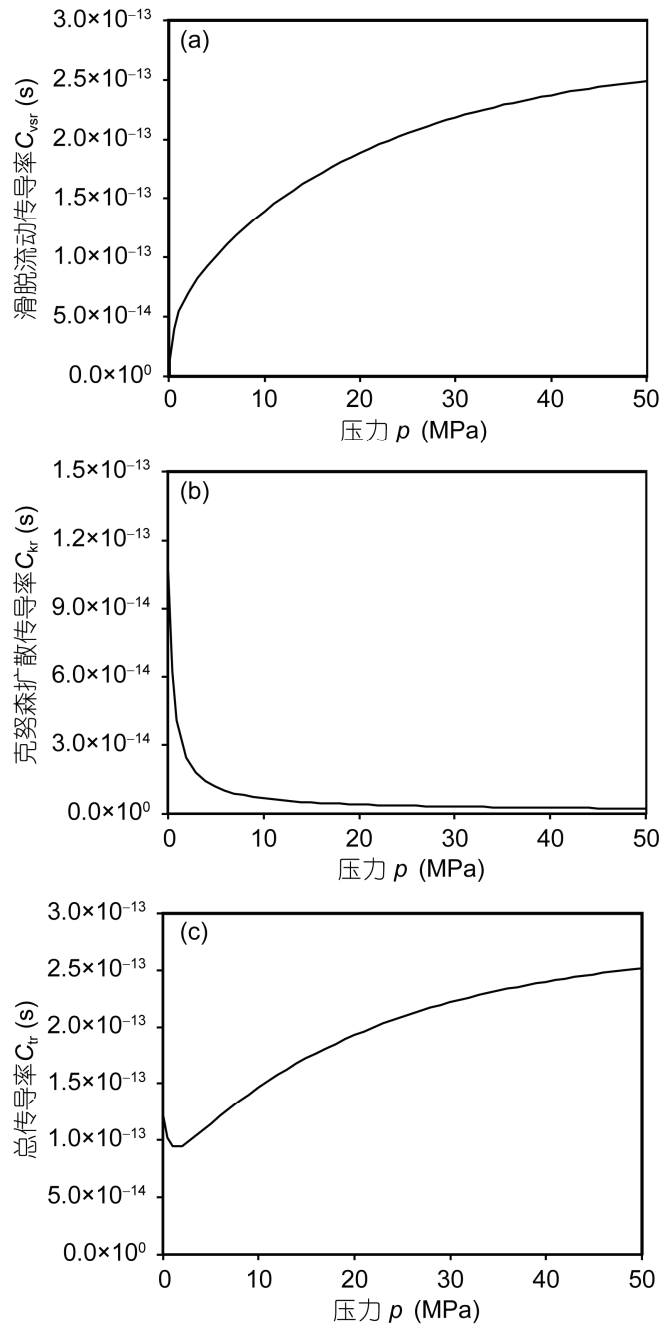

图 3 真实气体不同传导率关系曲线 $(r=5 \mathrm{~nm}, T=423 \mathrm{~K})$

积影响气体的传输, 尤其在高压条件下, 影响更加明 显. 这种真实气体效应可通过真实气体和理想气体 的分子自由程和不同传输量的偏差程度进行分析.

\subsection{1 平均分子自由程}

结合(1)式和(8) (10)式, 真实气体平均分子自由 程与理想气体平均分子自由程的比值可表达为

$$
\begin{aligned}
R_{\mathrm{r}}= & \frac{\lambda_{\mathrm{r}}}{\lambda_{\mathrm{r}}}=\left[1+\frac{A_{1}}{T_{\mathrm{r}}^{5}}\left(\frac{p_{\mathrm{r}}^{4}}{T_{\mathrm{r}}^{20}+p_{\mathrm{r}}^{4}}\right)+A_{2}\left(\frac{p_{\mathrm{r}}}{T_{\mathrm{r}}}\right)^{2}\right. \\
& \left.+A_{3}\left(\frac{p_{\mathrm{r}}}{T_{\mathrm{r}}}\right)\right] \sqrt{1+\frac{p_{\mathrm{r}}}{10.24 T_{\mathrm{r}}}\left[2.16 \frac{1}{T_{\mathrm{r}}}\left(\frac{1}{T_{\mathrm{r}}}+1\right)-1\right],}
\end{aligned}
$$

式中, $R_{\mathrm{r}}$ 是真实气体平均分子自由程与理想气体平均 分子自由程的比值, 无因次. 
(19)式表明比值 $R_{\mathrm{r}}$ 是对比压力和对比温度的函 数. $R_{\mathrm{r}}$ 随对比压力的增大而增大, 随对比温度的降低 而增大, 且越靠近临界温度区域 $\left(1.0 \leqslant T_{\mathrm{r}} \leqslant 1.6\right)$, 随对比 压力增大的幅度越大(图 4). 这是由于温度越低, 压 力越大, 气体密度越大, 气体分子之间的相互作用力 越强, 气体分子自身体积占纳米孔总体积的份额越 大, 因此, 真实气体效应对气体平均分子自由程的影 响就越显著.

\subsection{2 不同传输量}

为了探讨真实气体效应对纳米孔气体传输的影 响, 对真实气体传输模型和理想气体传输模型计算 的传输能力偏差程度进行分析.

纳米孔中的气体传输, 不论是滑脱流动、克努森 扩散, 还是所有的气体传输机理, 真实气体效应对气 体传导率影响都很大, 且随对比压力的增大而普遍 增大 $\left(p_{\mathrm{r}}>2.0\right.$ 且 $T_{\mathrm{r}}<1.4$ 区域除外), 随对比温度的降低 而增大(图 5). 这与真实气体效应对平均分子自由程 的影响类似, 压力越大, 温度越低, 气体密度越大, 气体分子之间的相互作用力越强，对气体传输的影 响越明显. 但当对比压力较大且对比温度较低 $\left(p_{\mathrm{r}}>2.0\right.$ 且 $\left.T_{\mathrm{r}}<1.4\right)$ 时，气体密度很大，此条件下气体分子自身 体积占纳米孔体积的份额变得越来越大, 其降低了 真实气体的传输能力, 且随压力增大, 降低幅度越大, 甚至超过因气体分子相互作用力而增大的传输能力. 所以, 表现为随压力的增大, 真实气体效应的影响反 而减弱.

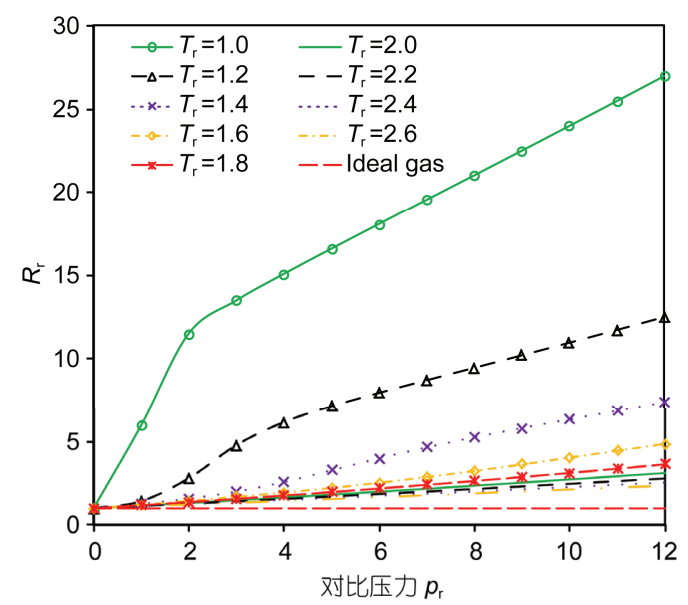

图 4 (网络版彩图) 不同对比温度下的真实与理想气体 分子自由程比值与对比压力关系曲线
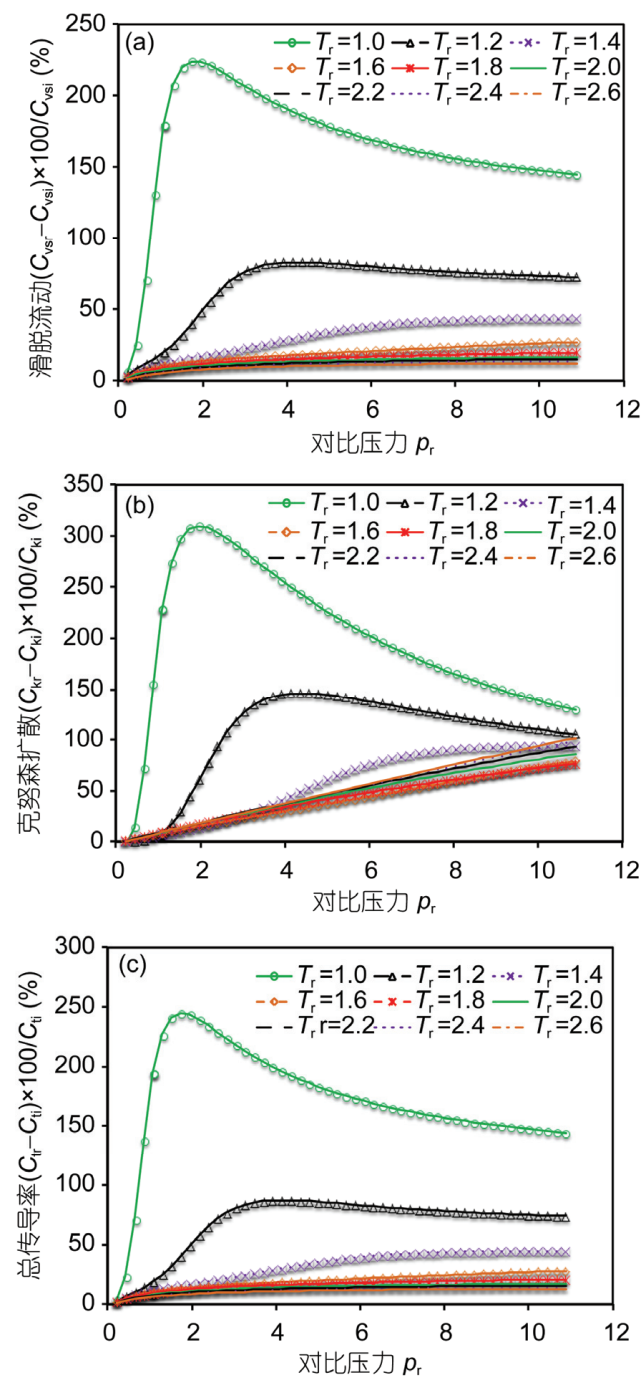

图 5 (网络版彩图)不同传输量偏差程度对比曲线 $(r=5 \mathbf{n m})$

\section{3 敏感性分析}

纳米孔气传输除了受压力和温度的影响外, 还 与纳米孔尺度和气体类型有关.

\subsection{1 纳米孔尺度}

相同压力下，纳米孔尺度越小，理想气体克努森 数与真实气体克努森数的差异越大, 表明真实气体 效应对气体传输影响越大(图 6). 温度越低, 影响程 度增大(图 6(b)), 即与页岩储集层相比，室内实验条 件下的真实气体效应对压力更加敏感.

以甲烷偏差程度 $5 \%$ 作为等值线, 做压力与温度 的交会图(图 7). 从图 7 可见, 温度相同时, 纳米孔隙 半径越小, 偏差程度 $\geqslant 5 \%$ 区域所对应的压力下限值 

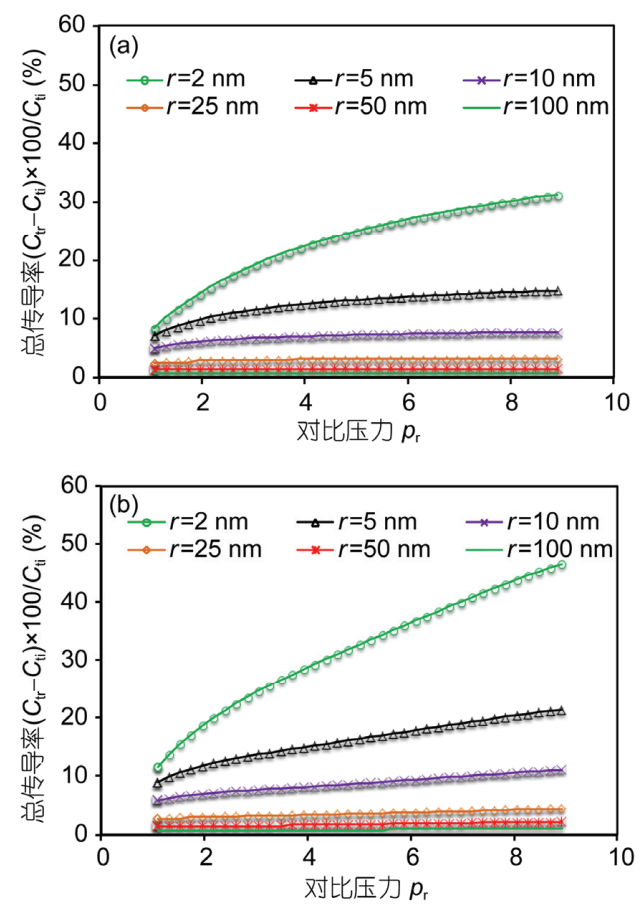

图 6 (网络版彩图) 不同纳米孔尺度的真实气体效应 (a) $T=423 \mathrm{~K}$ (页岩储集层温度); (b) $T=298 \mathrm{~K}$ (室内实验温度)

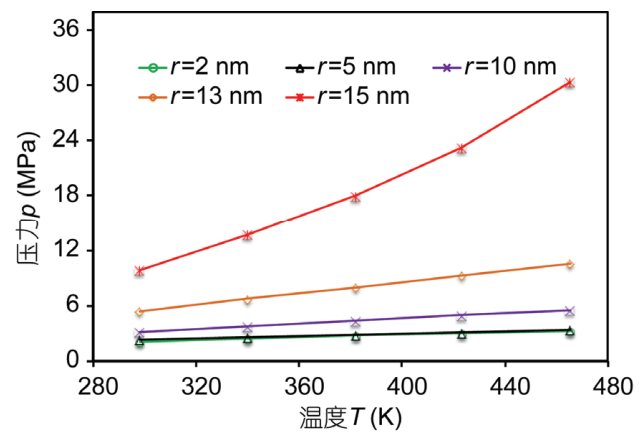

图 7 (网络版彩图) 不同纳米孔隙半径下甲烷偏差程度为 $5 \%$ 的等值线

越小; 当压力相同时, 纳米孔隙半径越小, 偏差程度 $\geqslant 5 \%$ 区域所对应的温度上限值越大. 这表明纳米孔隙 半径越小, 真实气体效应的影响范围越广.

\subsection{2 气体类型}

不同气体具有不同属性，包括气体摩尔质量、气 体分子大小、形状、极性等, 宏观上体现为具有不同 气体黏度、临界压力和临界温度, 因此具有不同气体 传输能力, 且真实气体效应的影响程度也不尽相同. 甲烷是页岩气的主要成分, 室内测页岩基质渗透率
实验的流体多为氮气和氦气，因此选取甲烷、氮气和 氦气 3 种气体进行真实气体效应敏感性分析，其影响 气体传输的属性参数见表 2 .

图 8 为 3 种不同气体压力与对比压力、温度与对 比温度关系曲线. 压力相同时, 对比压力大小依次为 氦气 >氮气 >甲烷(图 8(a)); 温度相同时, 对比温度大 小也依次为氦气 $>$ 氮气 $>$ 甲烷(图 8(b)). 对比压力越大, 气体分子之间的相互作用力越强，真实气体效应的 影响将越明显; 对比温度越大, 气体分子之间的相互 作用力越弱, 真实气体效应的影响也将越弱. 因此, 在同一温度和压力系统，甲烷、氮气和氦气的真实气 体效应影响程度的强弱是不同的, 需要具体分析.

在页岩储集层温度 $(423 \mathrm{~K}$ )和低压(小于等于 22 $\mathrm{MPa}$ )条件下, 真实气体效应主要受对比温度的影响, 因此，甲烷的真实气体效应影响程度最大；高压(大 于 $22 \mathrm{MPa}$ )条件下, 对比压力逐渐增大, 真实气体效

\section{表 23 种气体属性参数表}

\begin{tabular}{ccccc}
\hline 气体 & $\begin{array}{c}\text { 摩尔质量 } \\
\left.(\mathrm{g} \mathrm{mol})^{-1}\right)\end{array}$ & $\begin{array}{c}\text { 理想气体黏度 } \\
(\mathrm{Pa} \mathrm{s})\end{array}$ & $\begin{array}{c}\text { 临界压力 } \\
(\mathrm{MPa})\end{array}$ & $\begin{array}{c}\text { 临界温度 } \\
(\mathrm{K})\end{array}$ \\
\hline 甲烷 & 16.04 & $1.12 \times 10^{-5}$ & 4.5992 & 190.564 \\
氮气 & 28.01 & $1.78 \times 10^{-5}$ & 3.3958 & 126.192 \\
氦气 & 4.003 & $1.98 \times 10^{-5}$ & 0.22746 & 5.1953 \\
\hline
\end{tabular}
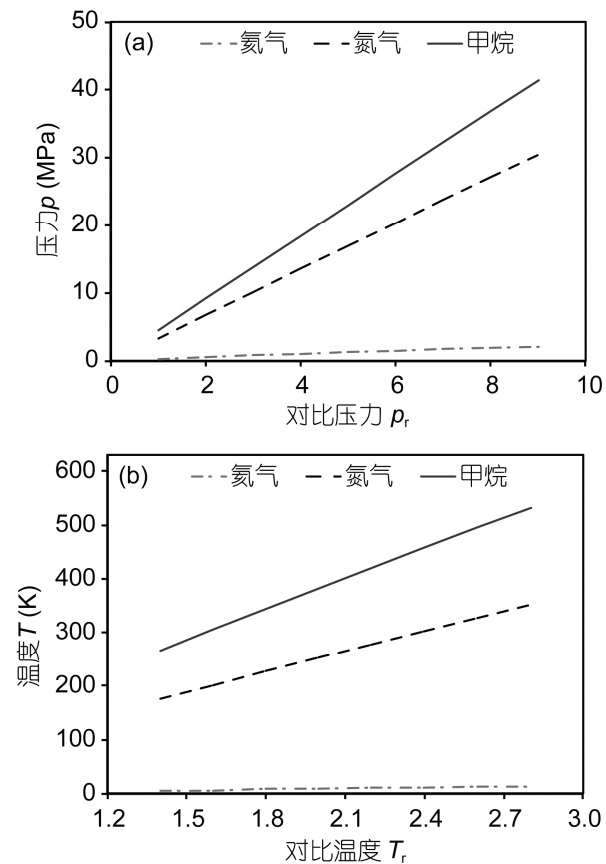

图 83 种不同气体压力与对比压力、温度与对比温度关系 曲线 
应受对比压力的影响也逐渐增大并占据主导作用, 此时氦气的真实气体效应影响程度最大(图 9(a)). 与 页岩储集层条件相比, 室内实验温度 $(298 \mathrm{~K})$ 较靠近 气体的临界温度, 因此对比温度主要控制了真实气 体效应的影响程度. 3 种气体中, 室内实验温度下甲 烷对比温度 1.56 最小, 且小于 1.6 , 较靠近临界温度 区域(结合图 4 的分析结果), 因此甲烷在全压力范围 内的真实气体效应影响程度都最大(图 9(b)).

以不同气体偏差程度 $5 \%$ 作为等值线, 分别做页 岩储集层条件下和室内实验条件下的压力与纳米孔 尺度的交会图版(图 10). 从图 10(a)可看出, 在页岩储 集层条件下, 当纳米孔尺度小于 $12 \mathrm{~nm}$ 时, 偏差程度 $\geqslant 5 \%$ 的区域所对应的压力下限最小的是甲烷, 表明 甲烷真实气体效应影响程度对压力最敏感; 当纳米 孔尺度大于 $12 \mathrm{~nm}$ 时, 偏差程度 $\geqslant 5 \%$ 的区域所对应的 压力下限最小的是氦气, 表明氦气真实气体效应影 响程度对压力最敏感. 综上所述, 纳米孔尺度还影响 了不同气体的真实气体效应影响程度的排序. 从图 10(b) 可看出, 在室内实验条件下, 甲烷真实气体效 应影响程度对压力最敏感, 因此, 当用甲烷做页岩基 质传导率测试实验时, 需考虑真实气体效应的影响, 即理想气体模型和真实气体模型计算的偏差.

不同气体具有不同的属性, 因此具有不同的气
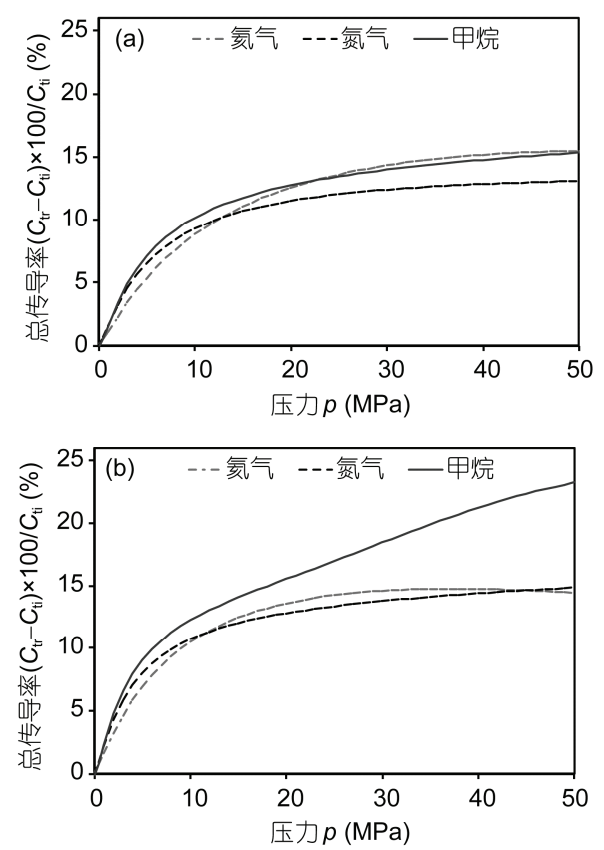

图 9 不同气体总传导率与压力的关系 $(r=5 \mathbf{n m})$ (a) $T=423 \mathrm{~K}$ (页岩储集层温度); (b) $T=298 \mathrm{~K}$ (室内实验温度)
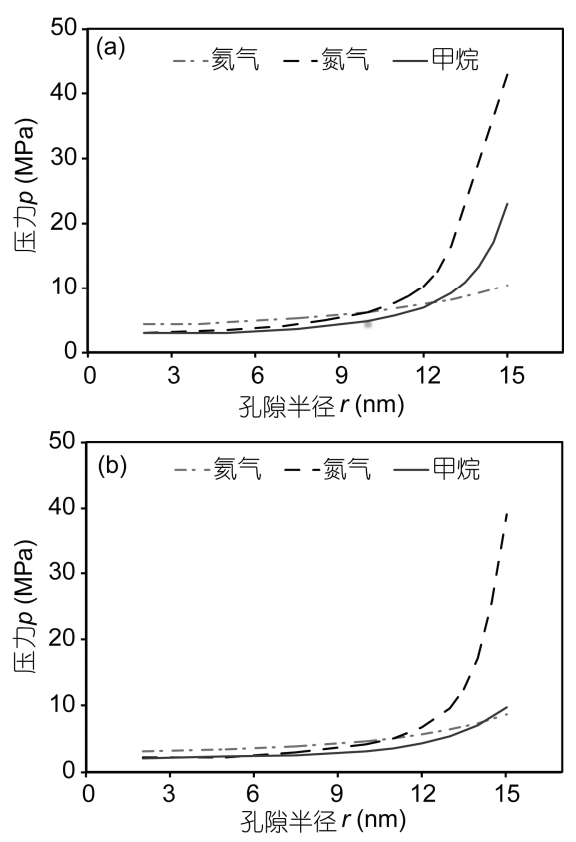

图 10 不同气体偏差程度 $5 \%$ 等值线对比

(a) $T=423 \mathrm{~K}$ (页岩储集层温度); (b) $T=298 \mathrm{~K}$ (室内实验温度)

体传输能力. 不论在页岩储集层条件下, 还是在室内 实验条件下, 如果用其他气体(氮气和氦气)模拟的传 输能力不经过校正, 直接应用到页岩气(主要成分甲 烷)的传输能力模拟中, 将出现偏差. 用氦气代替甲 烷，则低估了甲烷的传输能力; 而用氮气代替甲烷， 则高估了甲烷的传输能力(图 11). 与页岩储集层条件 相比, 室内实验条件下, 用氦气代替甲烷, 被低估的 传输能力减弱, 但仍可高达 $65.09 \%$; 而用氮气代替 甲烷，被高估的传输能力增大，可高达 $106.27 \%$. 因 此, 在用其他气体做室内页岩基质传导率测试实验 时, 需经过校正, 才能获得甲烷(页岩气)的真实传输 能力.

\section{5 结论}

基于滑脱流动和克努森扩散两种传输机理, 分 别以分子之间碰撞频率和分子与壁面碰撞频率占总 碰撞频率的比值作为滑脱流动和克努森扩散的权重 系数, 耦合这两种机理, 建立了理想气体传输模型. 同时考虑纳米孔高压条件下真实气体分子间相互作 用力和气体分子自身体积对气体传输的影响, 建立 了页岩纳米孔真实气体传输模型. 模型可靠性通过 已发表的分子模拟结果验证, 并表明纳米孔真实气 

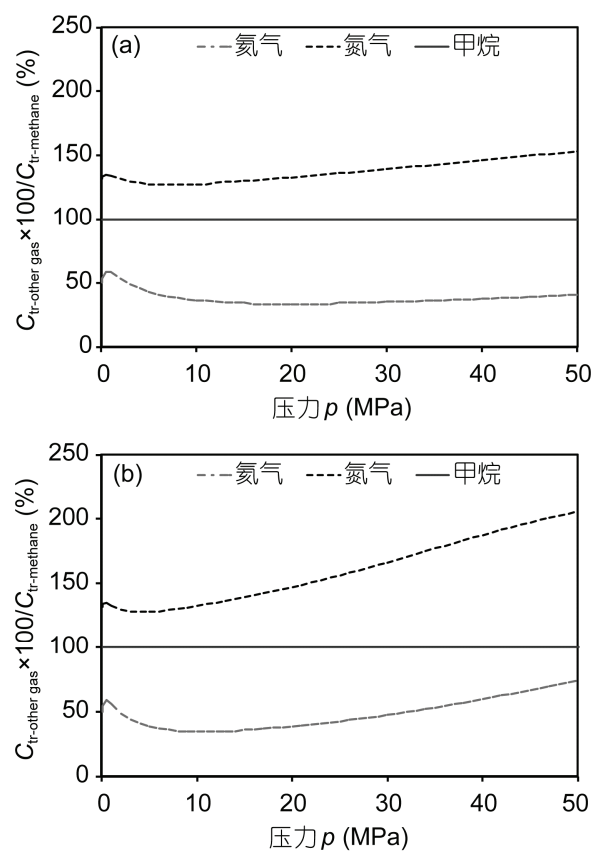

图 11 气体传导比与压力的关系 $(r=5 \mathrm{~nm})$ (a) $T=423 \mathrm{~K}$ (页岩储集层温度); (b) $T=298 \mathrm{~K}$ (室内实验温度)

体传输模型能够更合理地描述页岩纳米孔气体所有 的传输机理, 包括连续流动、滑脱流动和过渡流动. 模拟结果表明, 随压力降低, 纳米孔气体总传导率先 降低后增大, 这也是页岩气井开发具有很长稳产期 的原因之一.

压力越大, 温度越低, 气体密度越大, 气体分子
之间的相互作用力越强, 真实气体效应对气体传输 的影响越明显; 相同温度和压力条件下, 纳米孔尺度 越小, 真实气体效应对气体传输影响更大.

甲烷、氮气和氦气 3 种气体, 在储集层温度和低 压 $(p \leqslant 22 \mathrm{MPa})$ 条件下, 甲烷的真实气体效应影响程度 最大，可高达 $12.76 \%$; 高压 $(p>22 \mathrm{MPa})$ 条件下，氦气 的真实气体效应影响程度最大，可高达 $15.55 \%$; 在 室内实验条件下，甲烷真实气体效应的影响最大，可 高达 $23 \%$, 氮气和氦气可高达 $15 \%$, 也不可忽略. 因 此, 在模拟纳米孔气体传输时, 需考虑真实气体效应 的影响.

不同气体具有不同的属性，因此具有不同的气 体传输能力. 用氦气代替甲烷, 则低估了甲烷的传输 能力; 用氮气代替甲烷, 则高估了甲烷的传输能力. 与页岩储集层条件相比, 室内实验条件下, 用氦气代 替甲烷, 低估传输能力的程度减弱, 但仍可高达 65.09\%; 用氮气代替甲烷，高估传输能力的程度增大, 可高达 $106.27 \%$. 因此, 不论在页岩储集层条件下, 还是在室内实验条件下, 如果用其他气体(氮气和氦 气)模拟的传输能力, 都需要校正, 才能应用到页岩 气(主要成分甲烷)的传输能力模拟中.

值得注意的是, 页岩纳米孔壁面气体吸附/解吸 附, 仅是气体赋存状态变化(自由气和吸附气的相互转 换), 本身对页岩纳米孔气体传输并无贡献, 但影响纳 米孔壁面吸附气厚度, 从而影响页岩纳米孔气体的 传输. 本文忽略了此影响, 后续将进一步研究.

\section{参考文献}

1 Gao X C, Diniz da Costa J C, Bhatia S K. Adsorption and transport of gases in a supported microporous silica membrane. J Membr Sci, 2014, 460: 46-61

2 Michel G G, Sigal R F, Civan F, et al. Parametric investigation of shale gas production considering nano-scale pore size distribution, formation factor, and non-Darcy flow mechanisms. In: SPE Annual Technical Conference and Exhibition, 30 October-2 November, Denver, Colorado, USA, 2011. SPE 147438-MS

3 Ma J S, Sanchez J P, Wu K J, et al. A pore network model for simulating non-ideal gas flow in micro- and nano-porous materials. Fuel, 2014, 116: 498-508

4 Loucks R G, Reed R M, Ruppel S C, et al. Morphology, genesis, and distribution of nanometer-scale pores in siliceous mudstones of the Mississippian Barnett shale. J Sediment Res, 2009, 79: 848-861

5 Curtis M E, Sondergeld C H, Ambrose R J. Microstructural investigation of gas shales in two and three dimensions using nanometer-scale resolution imaging. AAPG Bull, 2012, 96: 665-677

6 Xiong X Y, Devegowda D, Michel G G, et al. A fully-coupled free and adsorptive phase transport model for shale gas reservoirs including non-darcy flow effects. In: SPE Annual Technical Conference and Exhibition, 8-10 October, San Antonio, Texas, USA, 2012. SPE 159758-MS

7 Tinni A, Fathi E, Agarwal R, et al. Shale permeability measurements on plugs and crushed samples. In: SPE Canadian Unconventional 
Resources Conference, 30 October-1 November, Calgary, Alberta, Canada, 2012. SPE 162235-MS

8 Civan F, Devegowda D, Sigal R. Critical evaluation and improvement of methods for determination of matrix permeability of shale. In: SPE Annual Technical Conference and Exhibition, 30 September-2 October, New Orleans, Louisiana, USA, 2013. SPE 166473-MS

9 Wu K L, Li X F, Wang C C, et al. Apparent permeability for gas flow in shale reservoirs coupling effects of gas diffusion and desorption. In: Unconventional Resources Technology Conference (URTEC) held in Denver, Colorado, USA, 25-27 August 2014. doi: 10.15530/urtec-2014-1921039

10 Klinkenberg L J. The permeability of porous media to liquids and gases. Drilling and Production Practice, 1 January, New York, 1941. 200-213

11 Mason E A, Malinauskas A P, Evans III R B. Flow and diffusion of gases in porous media. J Chem Phys, 1967, 46: 3199-3216

12 Beskok A, Karniadakis G E. A model for flows in channels, pipes, and ducts at micro and nano scales. Microscale Therm Eng, 1999, 3 : 43-77.

13 Javadpour F. Nanopores and apparent permeability of gas flow in mudrocks (shales and siltstone). SPE J Canadian Pet Tech, 2009, 48: $16-21$

14 Anderson J M, Moorman M W, Brown J R, et al. Isothermal mass flow measurements in microfabricated rectangular channels over a very wide Knudsen range. J Micromech Microeng, 2014, 24: 1-12

15 Curtis J B. Fractured shale-gas systems. AAPG Bull, 2002, 86: 1921-1938

16 Wang M R, Li Z X. Micro- and nanoscale non-ideal gas Poiseuille flows in a consistent Boltzmann algorithm model. J Micromech Microeng, 2004, 14: 1057-1063

17 Wang M R, Li Z X. An Enskog based Monte Carlo method for high Knudsen number non-ideal gas flows. Comput Fluids, 2007, 36: $1291-1297$

18 Wang M R, Lan X D, Li Z X. Analyses of gas flows in micro- and nano-channels. Int J Heat Mass Transfer, 2008, 51: 3630-3641

19 Civan F, Rai C S, Sondergeld C H. Shale-gas permeability and diffusivity inferred by improved formulation of relevant retention and transport mechanisms. Transp Porous Media, 2011, 86: 925-944

20 Javadpour F, Fisher D, Unsworth M. Nanoscale gas flow in shale sediments. J Can Petrol Technol, 2007, 46: 55-61

21 Karniadakis G E, Beskok A, Aluru N. Microflows and Nanoflows: Fundamentals and Simulation. New York: Springer-Verlag, 2005

22 Darabi H, Ettehad A, Javadpour F. Gas flow in ultra-tight shale strata. J Fluid Mech, 2012, 710: 641-658

23 Choi J G, Do D D, Do H D. Surface diffusion of adsorbed molecules in porous media: Monolayer, multilayer, and capillary condensation regimes. Ind Eng Chem Res, 2001, 40: 4005-4031

24 Loebenstein W V. Calculations and comparisons of nonideal gas corrections for use in gas adsorption. J Colloid Interface Sci, 1971, 36: 397-400

25 Jarrahian A, Heidaryan E. A simple correlation to estimate natural gas viscosity. J Nat Gas Sci Eng, 2014, 20: 50-57

26 Civan F. Effective correlation of apparent gas permeability in low-permeability porous media. Trans Porous Media, 2010, 82: 375-384

27 Wei Y S, Sadus R J. Equations of state for the calculation of fluid-phase equilibria. AIChE J, 2000, 46: 169-196

28 Gao X C, Diniz da Costa J C, Bhatia S K. The transport of gases in a supported mesoporous silica membrane. J Membr Sci, 2013, 438: $90-104$

29 Loyalka S, Hamoodi S. Poiseuille flow of a rarefied gas in a cylindrical tube: Solution of linearized Boltzmann equation. Phys Fluids A, 1990, 2: 2061-2065 


\title{
Real gas transport through nanopores of shale gas reservoirs
}

\author{
WU KeLiu ${ }^{1,2}$, LI XiangFang ${ }^{1} \&$ CHEN ZhangXin ${ }^{2}$ \\ ${ }^{1}$ School of Petroleum Engineering, China University of Petroleum, Beijing 102249, China; \\ ${ }^{2}$ Department of Chemical and Petroleum Engineering, University of Calgary, Calgary T2N1N4, Canada
}

The gas transport in nanopores of shale gas reservoirs significantly differs from that in conventional gas reservoirs. A model for ideal gas in nanopores is derived based on a weighted summation of slip flow and Knudsen diffusion, where ratios of molecule-molecule collisions and molecule-wall collisions to total collisions are the weighted factors of slip flow and Knudsen diffusion, respectively. This model is extended to the application of real gas transport in nanopores, taking into account the effects of molecule-molecule force and gas molecule volume on mass transport under the condition of high pressure. The model reliability is validated by published molecular simulation results. The results show that the model can be more reasonable to describe all of the gas transport mechanism known, including continuous flow, slip flow and transition flow; the real gas effect on gas transport is up to $23 \%$, which is affected by pressure, temperature, nanopores radius and gas type; and transport capacity is underestimated by $65.09 \%$ with helium and overestimated by $106.27 \%$ with nitrogen in modeling methane transport in shale nanopores under the condition of laboratory experiments.

shale gas reservoirs, nanopores, real gas, slip flow, Knudsen diffusion

doi: 10.1360/N092015-00076 